\title{
RESOURCE-SAVING AND RELIABILITY OF TRANSPORTATION SYSTEMS
}

\author{
Tkhoruk E.I.
}

National University of Water and Environmental Engineering (NUWEE), Associate Professor, Candidate of Technical Science, Associate Professor, Department of Transport Technologies and

Technical Service, Ukraine

\section{Krystopchuk M.E.}

National University of Water and Environmental Engineering (NUWEE), Associate Professor, Candidate of Technical Science, Associate Professor, Department of Transport Technologies and Technical Service, Ukraine

\section{Soroka V.S.}

National University of Water and Environmental Engineering (NUWEE), Associate Professor, Candidate of Agricultural Sciences, Associate Professor, Department of Transport Technologies and Technical Service, Ukraine

\section{Holotiuk M.V.}

National University of Water and Environmental Engineering (NUWEE), Associate Professor, Candidate of Technical Science, Associate Professor, Department of Transport Technologies and Technical Service, Ukraine

\footnotetext{
Abstract

The article is based on the analysis of the development of the theory of reliability and its indicators are formulated indicators of the reliability of transport systems and built a system of factors determining their reliability. It is established that the formation of the theory of reliability of transport systems today is not completed. Based on the specifics of the systems, new reliability indicators were proposed and substantiated such as emergency downtime and reliability of emergency downtime. On the basis of the performed studies, it was found that the specifics of the transport process require the introduction of emergency downtime close to the recovery time and the reliability of emergency downtime. It is necessary the introduction of two close parameters such as the average recovery time and the average emergency idle time. They are associated with the need to distinguish between the idle time of a technological system or a separate element when a failure occurs and the idle time is need-
} 
ed. For the technological links of the transport system, the main purpose of which is to ensure efficient operation transport process, the probability of emergency downtime can serve as a criterion for assessing their reliability.

\section{Introduction}

The problem of reliability of transport systems is one of the main ensuring and improving the efficiency of their functioning. The reliability parameter of the transport system is one of the main indicators of the quality and efficiency of its operation, it manifests itself in time and reflects changes occurring in the system during the whole period of its operation. To date, the situation with the assessment and ensuring of the reliability of transport by road significantly impedes the achievement of the transport system indicators of transport strategy indicated in the Transport Strategy. The quality indicator in transport offered by scientists today is reliability taking into account mainly only the reliability of technical means, while reliability of performed services is practically not considered. There is also no standardization of reliability in road transport and in reports of motor transport enterprises there are no criteria characterizing reliability and efficiency of their work. The formation of the theory of reliability of transport systems is not completed to date. Therefore, the bulk of organizational and technological decisions to reduce the number of failures is based on the knowledge of the methodology for solving traffic safety problems, technical maintenance of vehicles, situational management of motor transport, the theory of reliability of technical systems, risk management, supply chain management and other scientific fields.

The quality of transport system operation is set by the consumer. Despite the large variety of indicators of quality of delivering goods and passengers by road, they are based on the same or similar parameters. It is appropriate to evaluate the quality of the transport system by its accessibility, functionality and reliability, as suggested by D. Baueroks and D. Kloss regarding to supply chains [18].

Based on this statement, reliability is one of the most important components of assessing the quality of the transport system. The established interconnection of efficiency, quality and reliability of transport system operation prevents these definitions, simplifies the choice of criteria of reliability estimation, formulation concepts of "reliability" and "failure", as well as the concept of "Reliability management of transport systems". 
In researches, that examin the problem of quantitative assessment of the reliability of cargo delivery systems, usually is implemented an approach based on the use of terminology and results in the field of ensuring the reliability of technical facilities and conducting appropriate analogies. In technology, reliability includes, among other properties, failure-freeness [19]. Reliability is considered satisfactory if the failures do not exceed the specified level. A failure is called a breakdown, disability, or event after which the operating parameters of the unit or machine go beyond acceptable limits. The reliability of the functioning of transport systems should be evaluated by the number of breaches of the contract for delivery. If there are no such violations when performing the transport service, then the reliability is at the highest possible level.

Thus, the reliability of transport systems is a complex feature that includes the ability of the transport system to meet the agreed upon between the customer and the service provider requirements for the amount and condition of the cargo being carried, the safety of passengers and their luggage, adherence to the schedule of the transport process, as well as support and restoring the specified level of transport service.

By analogy with the definition given, it would be logical to assert that the management of the reliability of transport systems for the delivery of goods and passengers by road involves the implementation of measures ensuring the level of reliability agreed by both sides, its support and further enhancement to the economically feasible limit.

Fundamental to the theory of reliability is the concept of "failure". This definition is based on the methodological apparatus of analysis and quantification of the reliability of objects, processes and systems, regardless of their complexity, purpose and scope.

In the theory of reliability of technical systems, failure is an moment after which the operating parameters of the unit, machine or process go beyond the permissible limits. This definition is valid for both simple technical objects and complex technical systems and technological processes [20]. By counting the number of such events per unit of time, you can estimate the reliability of the object.

Reliability will be satisfactory provided if the number of failures does not exceed the specified level. In developing this approach, the 
failure of the transport system can be defined as a failure to fulfill an accepted order (request) for delivery, as well as a violation of the agreed in the contract requirements for the quantity and quality of the goods being carried, the safety of passengers and luggage, the schedule of the transport process, the restoration of a given level of transport servicing [21].

The problem of reliability in transport has significant features and is not limited to the reliability of technical means. It also determined the conditions and laws of all elements of the transport process [15]. The main problems of the theory of reliability are to establish patterns of occurrence of failures and their recovery; determining the quantitative characteristics, methods of calculation of reliability. The most studied in this it is considered theory of reliability technical systems. Principles of reliability of technical objects are adapted to the conditions of reliability of supply chains [5], [17]. Reliability transport systems and road transport process has methodological connection with the reliability of technical systems since to improve the quality of delivery of cargo and passengers as well as in technique applied reservation. There are structural [11] and functional reservation [10], [12].

In reliability theory, in particular, processes occurrence of failures, recovery of production systems elements are described by methods of probability theory [16].

Pronnikov A.S., Druzhynin G.V. consider the reliability of the system based on determining the function of readiness system that consists of $n$ subsystems. In this case the function of readiness system describes the probability of finding the system in working condition at any time [13], [7].

The problem of reliability is complex both in terms of its characteristics and measurements, and its cause-effect displays. Thus the reliability of vehicles is based on the classic approach to reliability that investigates the reliability of all systems and mechanisms that in recent works called "mechanical reliability" [8], and the reliability of the technical maintenance and repair and reliability in the process of commercial exploitation also take into account organizational and economic factors. In reliability theory, considerable attention is given to the impact of damages from insufficient of reliability machines as an economic expression of technogenic risk [14]. Aulin V.V., Golub 
D.V engaged in issues of legal regulation to provide reliability of functioning transport systems in Ukraine [1], also they have investigated methods for assessing and analyzing the reliability of automobile transport systems [2], [3], [4].

Classical methods of quantitative assessment of indicators of reliability of complex systems [9] applied to systems for which the notion of failure is clearly identified, that system is in one of two states: in a state working or in a state of recovery.

The proposed in the article recommendations are based on the definitions of probability reliability indicators of transport systems.

\section{Materials and Methods}

The main tasks of reliability theory are the establishment of patterns of occurrence of failures and their recovery, determination of quantitative characteristics, development of methods of evaluation and calculation of reliability. There are two approaches to solving these problems. The first approach is to study statistical the patterns of failure of the same type of technical means in certain operating conditions and is the basis for the establishment of distribution laws of the investigated parameters and obtaining of their numerical characteristics operational reliability.

Found in this way specifications which are used for calculating the reliability of technological systems consist of the types of technical means for which they are identified.

The second approach is aimed at studying the physical nature and mechanism of failures. It serves as a basis for the development of measures to improve the reliability of existing and planned technological systems. This approach is more efficient in terms of identifying physical essence reasons which caused the deviations options below acceptable limits, which means rejection. This path is used where, despite specificity of operation is still possible to obtain the necessary input data, such as the study of the reliability of transport systems.

To obtain analytical expressions of reliability of a transport system, we assume that it operates according to the following scheme: subsystems and elements, which were rejected, begin to recover; there are no restrictions on the number of restorations; failure of one of the elements or subsystems will result in the refusal of the transport system as a whole. 
To evaluate the reliability of the elements of transport systems used probabilistic parameters of reliability.

The reliability of the functioning of transport systems and processes of road transport is a complex feature that includes the ability of the system to fulfill the requirements for the number and condition of the cargo carried, the safety of passengers and their luggage, compliance with the schedule of the transport process, as well as support and restoration of a given level of transport service.

One of the parameters of reliability is the probability of failure operation work. Failures in the transport system may be caused by some emergency situations in the functioning of its elements. Multitransport system can occur in three forms: while eliminating the consequences of the situation in the transport process, when combined parties of their functions, the redevelopment of activity of the participants of the transport process. Any emergency situation caused by the refusal arising in the performance of transportation usually leads to changes in the technology of work its members. Reliability of transport system depends on several factors. (fig. 1)

Some emergency situations in road transport are common, such as a traffic accident or technical malfunction of the vehicle on line [11].

The algorithm of actions and approximate duration of treatment elimination of consequences of the typical situations is standard and is known in advance. Work by the transport process in the mode of elimination of the accident is a reserve (auxiliary) function and work as usual is the main function. Multifunctionality is reflected in the form of performances and methods of solving problems for managing reservation reliability of the transport process and its components.

In this case, based on the fact that at any time $t$ state of the element or subsystem is described by the random vector

$$
\left.x(t)=\left[x_{1}(t), x_{2}(t)\right), \ldots x_{n}(t)\right]
$$

which can take two values always at the one-dimensional variable: $x(t)=1$, if the system or component is in working condition, and $x(t)=0$ in case of failure. The components of the vector $x(t)$ can be the values of various parameters of the system. 


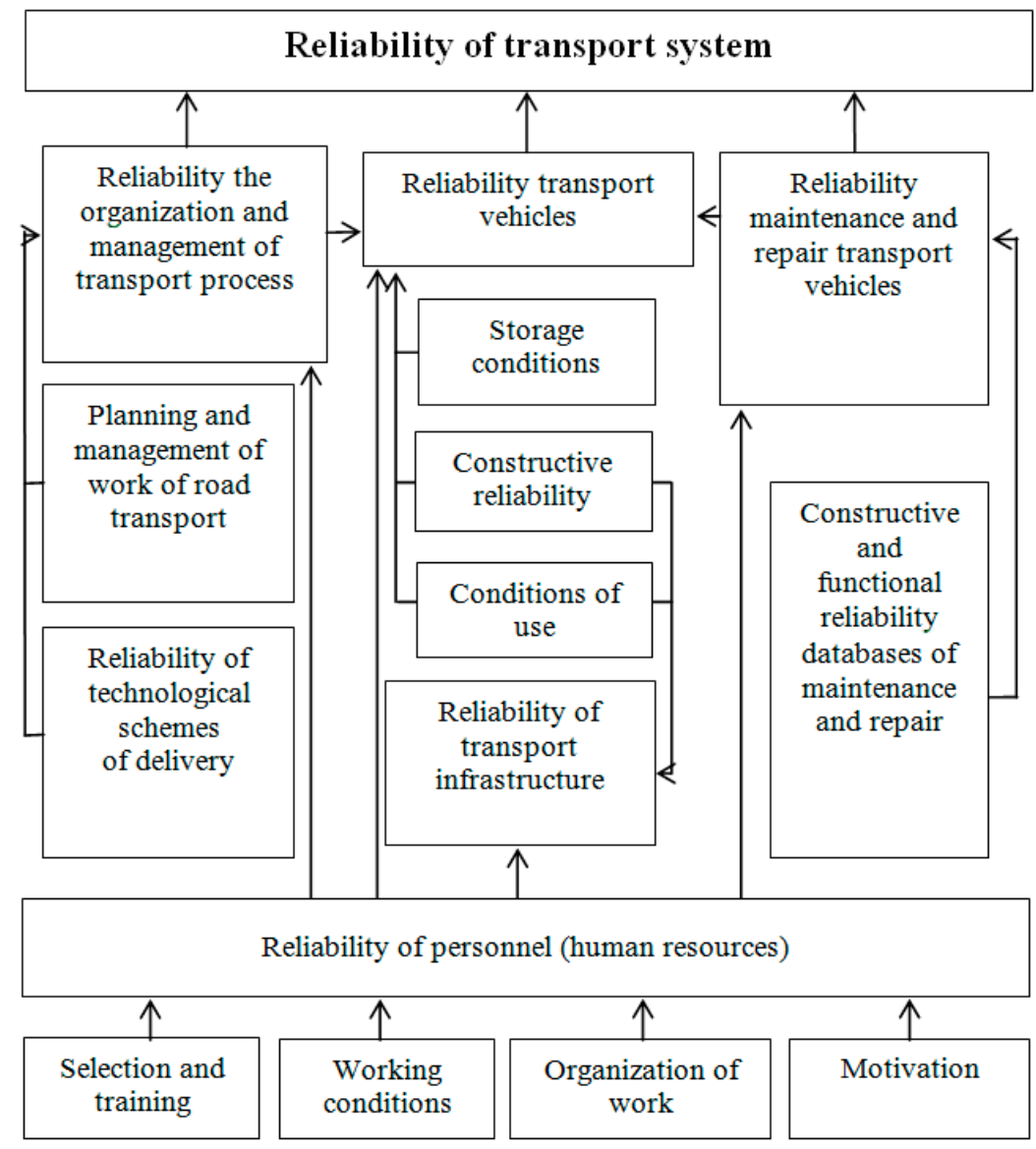

Fig. 1. Factors that determine the reliability of transport system

Random vector $x(t)$ is characterized by a probability distribution $F\left(x_{1}, \ldots, x_{2}, x_{\mathrm{n}}, t\right)$ i.e. the probability that the $x_{1}(t) \leq x_{1}, \ldots, x_{\mathrm{n}}(t) \leq x_{\mathrm{n}}$.

Each of the limit values that characterize the state of the system meets the mathematical expectation of the objective function $G(t)$ at the interval of time $a \leq x \leq b$.

Reliability of elements that make up the technological system, characterized probabilistic indicators (table 1), the most important of 
which are the uptime and recovery time. These options are probabilistic in nature and describe the relevant laws of distribution.

Indicators of reliability

\begin{tabular}{|c|c|c|}
\hline \multicolumn{3}{|c|}{ Recoverable elements } \\
\hline № & The name of indicator & Mathematical expression \\
\hline 1 & Average uptime & $T_{u}=\int P(t) d t$ \\
\hline 2 & Average recovery time & $T_{a r}=\int_{0}^{0} \tau d \nu(\tau)=\int_{0} G(\tau) d \tau$ \\
\hline 3 & Reliabilities & $P(t)=\operatorname{Bep}(T \geq t)$ \\
\hline 4 & Failure rates & $\lambda(t)=\frac{f(t)}{P(t)}=\frac{d \ln P(t)}{d t}$ \\
\hline 5 & Reliabilities for the time $(0, \tau)$ & $v(t)=\operatorname{Bep}\left(T_{B}\langle\tau)\right.$ \\
\hline 6 & $\begin{array}{l}\text { The probability of no recovery time }(0, \\
\tau)\end{array}$ & $G(\tau)=1-v(t)=\operatorname{Bep}\left(T_{B} \geq \tau\right)$ \\
\hline 7 & $\begin{array}{l}\text { The density of probability of recovery } \\
\text { the time } \tau\end{array}$ & $\nu(t)=V\langle\tau)$ \\
\hline 8 & $\begin{array}{l}\text { The intensity of recovery the time } \tau, \\
\tau_{i}-\text { the duration of recovery of } \mathrm{i} \text {-th } \\
\text { failure, } \\
n \text { - the number of failures }\end{array}$ & $\mu(\tau)=\frac{v(\tau)}{1-V(\tau)}, \quad \mu=\frac{n}{\tau_{i}}$ \\
\hline
\end{tabular}

Transport systems relating to renewable systems that are characterized by intervals periodically alternating correct operation and restore during the failure.

Because of this indicators of reliability is selected in such a way that they can be would be to assess the reliability of individual elements, and generally renewable systems consisting of different types of elements.

Indicator of reliability which taking into account the uptime $T_{u}$ and downtime $\tau_{d}$ is readiness coefficient

$$
K_{R}=\frac{T_{u}}{T_{u}+\tau_{d}}
$$

and the coefficient of failure

$$
K_{f}=1-K_{R}=\frac{\tau_{d}}{T_{u}+\tau_{d}}
$$


In fig. 2 presented the dependence of the readiness coefficient and the coefficient of failure of uptime and downtime consumer.

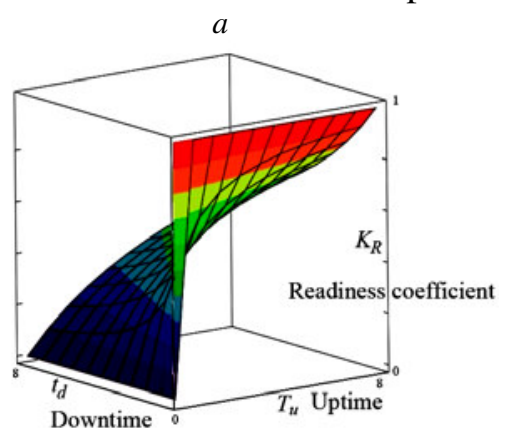

$b$

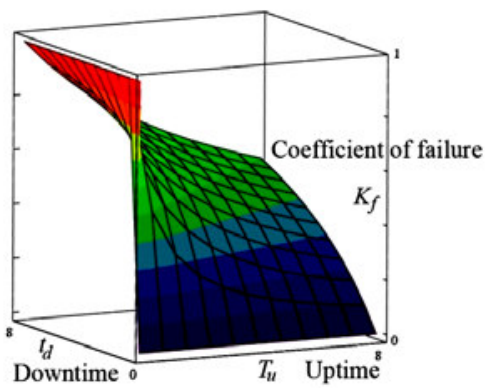

Fig. 2. Dependence of the readiness coefficient and the coefficient of failure of uptime and downtime: $a$ - dependence of the readiness coefficient of uptime and downtime; $b$ - dependence of the coefficient of failure of uptime and downtime

In view of the data table1 we get

$$
\begin{aligned}
& K_{R}= \frac{\int_{0}^{\infty} t \cdot f(t) d t}{\int_{0}^{\infty} t \cdot f(t) d t+\int_{0}^{\infty} \tau \cdot d V(\tau)} \\
& K_{f}=\frac{\int_{0}^{\infty} \tau \cdot d V(\tau)}{\int_{0}^{\infty} t \cdot f(t) d t+\int_{0}^{\infty} \tau \cdot d V(\tau)}
\end{aligned}
$$

i.e. two parameters are characterized by distribution law uptime and recovery time and reflect the probability of finding components or systems in working order or in a state of failure, respectively.

The relative time of consumer's downtime by reason of failure of the system over a period of work.

$$
\bar{P}(e)=\frac{t_{D}}{T_{c o}}=\frac{t_{D}}{t_{D}+T_{U P}}
$$

where: $t_{D}$ is downtime of consumer for over a period of work;

$T_{U P}$ is uptime for a certain period. 
Indicator $\bar{P}(e)$ of (6) is the probability of stimulated (emergency) downtime and form with a probability of a working state of complete group of events

$$
\bar{P}(e)+P(t)=1
$$

These terms define more precisely the probabilistic nature of the indicators of reliability. They take into account the frequency of occurrence of failure and downtime and represented more comfortable by using.

Any considered technological scheme can be represented as a system composed of a number of elements. If the i-th element of the system has been in operation for the time $T_{E}$ then this value consists of alternating time intervals, correct operation $t_{c o}$ and recovery time $T_{r}$. However, the recovery failure $T_{r}$ does not fully reflect the time of consumer in forced waiting for service. This specificity characteristic for the transport process requires the introduction of another indicator that comes close to recovery time, namely, time of emergency downtime $t_{e d}$.

Significantly it affects the type of the distribution of the random variable on the accuracy of results in calculations for reliability.

For example, the exponential distribution law uptime element or some technological system indicates that the flow of failures can be adopted the simplest.

Be noted the basic properties of the simplest flow failures:

$a$ - failure rate is constant ( $\lambda=$ const $)$ and average uptime

$$
T_{u}=\frac{1}{\lambda}
$$

$b$ - density of probability of time intervals between the neighboring failures

$$
f(t)=\lambda \cdot \exp (-\lambda t) f(t)=\lambda \cdot \exp (-\lambda t)
$$

$c$ - probability of getting $\mathrm{n}$ events in the time interval $[t, t+\Delta t]$ gets according to Poisson

$$
P_{n}=\frac{1}{n !}(\lambda t)^{n} \exp (-\lambda t) P_{n}=\frac{1}{n !}(\lambda t)^{n} \exp (-\lambda t)
$$

where $\lambda t$ is the average number of failures in an interval of duration $t$; 
$d$ - the probability of absence of failures in the interval duration $\tau$, which starts at a random time determined by the equation

$$
P_{0}=\exp (-\lambda t)
$$

\section{Results}

The simplest flow failures and recoveries in transport technological systems can be quite completely described by three numerical indicators of reliability, which are important to get to practical use. These characteristics are the average intensity of failures $\lambda_{a v}$, average recovery time $T_{a r}$ and the average emergency downtime $t_{a v}$. The introduction of two close parameters: the average recovery time $T_{a r}$ and the average emergency downtime $t_{a v}$ is associated with the necessity of the separation time finding in state of downtime technological system or a separate element with the appearance of failure and downtime of consumer.

Probability of emergency downtime defined by the formula

$$
\bar{P}(e)=\frac{t_{M E}}{t_{M E}+T_{c o}}
$$

where $t_{M E}$ is the mathematical expectation of time of forced (emergency) downtime, $t_{M E \approx} \approx t_{a e d}$;

$T_{c o}$ is the time of correct operation during the observation time;

$T_{c o}+t_{M E}=T_{o}$. is the observation time.

Or

$$
\bar{P}(e)=\frac{n \cdot t_{d}}{N \cdot T_{o}}=\lambda \cdot t_{d}=\frac{t_{d}}{T_{u}}
$$

where $n$ is the number of failures of elements of the system during the observation time $T_{\mathrm{o}}$;

$N$ is the number of elements of the system which are under the observation.

From (13) implies that the probability of emergency downtime represents size of relative of time of emergency downtime and therefore can be used to determine the economic damage caused by interruptions in the functioning of technological systems.

The probability of emergency downtime linked to probability of faultless work with the following ratio. With exponential distribution law uptime 


$$
P(t)=\exp (-\lambda t)=\exp \left(\frac{t}{T_{u}}\right)
$$

whence

$$
\lambda=-\frac{\ln P(t)}{t}, \quad \bar{P}(e)=\lambda t_{d}
$$

If the probability of faultless work $P(t)$ is given, then

$$
\bar{P}(e)=\lambda_{a v} t_{e d}
$$

\section{Discussion}

Thus, specificity which is typical for transport process requires the introduction time of emergency downtime $t_{e d}$, which is close to the time of recovery. This indicator can be attributed to one of the main numerical reliability features that characterize a simplest flow failures and recovery in transport technological systems.

The introduction of two close parameters: the average recovery time $T_{a r}$ and the average emergency downtime $t_{a e d}$ is associated with the necessity of the separation time finding in state of downtime technological system or a separate element with the appearance of failure and downtime of consumer.

For technological parts of the transport system, the main purpose of which consists in maintenance conditions for effective functioning of the transport process, the probability of emergency downtime can serve as a criterion for evaluating their reliability.

\section{Conclusions}

An important indicator to assess the reliability of renewable transport systems is the probability of an emergency downtime, which reflects both the frequency of failure and the idle time.

From the practical point of view, the above models allow us to determine the general probability of a forced idle or other reliability measure for virtually any scheme of the transport process, and on this 
basis to assess its reliability either in absolute value of the indicator, or with the help of economic expression of reliability in the form, for example, mathematical expectation of damage.

\section{References}

1. Aulin V.V., Golub D.V. 2016. Legal and Regulatory ensure the reliability of transport systems in Ukraine, Bulletin of Zhytomyr State Technological University. Series: Engineering Vol.2 (77), P. 28-33, Zhytomyr/ Ukraine.

2. Aulin V. V., Golub D. V. 2017. Methods of estimation and analysis of reliability of automobile transport systems. Book of abstract the International scientific and technical conference of young researchers and students "Current issues in modern technologies" (Tern., 16-17 November 2017), vol. 3, pp. 14-15 [in Ukrainian]

3. Aulin V.V., Holub D.V., Hrynkiv A.V., Lysenko S.V. Methodological substantiation of the research and the solution problems of reliability of transport system operations: monograph, Kropyvnytskyi: Vydavnytstvo TOV "KOD", 2017, $370 \mathrm{p}$.

4. Aulin V., Golub D., Hrynkiv A., Lysenko S. Methodological substance of research and solution of problem of reliability of vehicle functioning/ Technical service of agriculture, forestry and transport systems №10 (2017), P. 29-36

5. Bochkaryov A. A., Bochkaryov P. A. 2010. Problem of the reliability of the supply chain, Logistics: modern trends of development: Proceedings of the IX Intern. scientific. - pract. Conf., SPbGIEU, P. 64-67., St. Petersburg /Russia.

6. Boyko A.I., Bondarenko O.V., Savchenko V.M. 2013. The mathematical model's fundamental matrix of active redundant model's reliability derivation, Federal interdepartmental scientific and technical collection. Design, production and operation of agricultural machinery, Vol.2(43), №2(43)), KNTU, P. 49-54, Kirovograd / Ukraine.

7. Druzhinin G.V. 1978. Reliability of automated systems, Energy, 336 p., Moscow/Russia.

8. Grinchenko A.S. 2012. Mechanical reliability of mobile machines. Evaluations, simulation, control, Apostrophe, 259 p., Kharkiv / Ukraine.

9. Ignatov V.I. 2000. Scientific bases of formation of strategy of maintenance and repair of forest machinery, MGUL, $336 \mathrm{p}$., Moscow/Russia. 
10. Kurganov V.M., Gryaznov M.V. 2012. Ensuring of reliability in the traffic management system and the production of road transport: monograph, House of Press, 128 p., Magnitogorsk/Russia.

11. Kurganov V.M., Gryaznov M.V. 2013. Managing reliability of transport systems and processes of automobile transportations: monograph, House of Press, 318 p., Magnitogorsk/Russia.

12. Kurganov V.M., Gryaznov M.V. 2014. Structural redundancy in auto motive transport, World of Transport and Transportation, Vol.5, P. 6-21, Moscow/Russia.

13. Pronnikov A.S. 1978. Reliability of machines, Mechanical engineering, 234 p., Moscow/Russia.

14. Repin S.V. 2008. Methodology of improvement of the system of the technical operation of construction machinery: Dis. dts, 451 p., St. Petersburg /Russia.

15. Rezer S.M. 1994. Transport management abroad, The science, 315 p., Moscow/Russia.

16. Ventsel E.S. Ovcharov L.A. 1983. Applied tasks of Probability Theory, Radio and Communications, 416 p., Moscow/Russia.

17. Zaytsev Y.I. 2008. The problem of reliability in the supply chain process model, Logistics and Supply Chain Management: current trends in Russia and Germany, Coll. articles of the Russian-German conference DRLOG, Publishing House Polytechnic University, P. 266-271, St. Petersburg /Russia.

18. Belikov A.Yu. Risk Theory. Textbook. manual / A.Yu. Belikov, Education of the Russian Federation. - Irkutsk: Irkutsk Publishing House. state. econom. Acad. 2001. $-95 \mathrm{p}$.

19. DSTU 2860-94. Reliability of technology. Terms and definitions.

20. International Electrotechnical Dictionary. Reliability and quality of services. IEC Publication 50 (191): Per. from English: M: MNTK "Reliability of machines". 1990. 66 p.

21. Kurganov V.M., Gryaznov MV. Terminology in the sphere of providing of transport systems and transportation processes dependability / Proceedings of the 8th International Scientific and Practical Conference "Key Issues in Modern Science", 2012. Volume 32. Technology. Sofia. White City-BG Ltd. - P 28-34. 